\title{
A closure model for turbulent convection. Application to the excitation of $p$ modes
}

\author{
K. Belkacem ${ }^{1}$, R. Samadi ${ }^{1}$, M.-J. Goupil ${ }^{1}$, F. Kupka ${ }^{2}$ and F. Baudin ${ }^{3}$ \\ ${ }^{1}$ Observatoire de Paris, LESIA, CNRS UMR 8109, 92195 Meudon, France \\ email: kevin.belkacem@obspm.fr \\ ${ }^{2}$ Max-Planck-Institute for Astrophysics, Karl-Schwarzschild Str. 1, 85741 Garching, Germany \\ ${ }^{3}$ Institut d'Astrophysique Spatiale, CNRS/Université Paris XI UMR 8617,
} 91405 Orsay Cedex, France

\begin{abstract}
Oscillations of stellar $p$ modes excited by turbulent convection are investigated. In the uppermost part of the solar convection zone, radiative cooling is responsible for the formation of turbulent plumes, hence the medium is characterized with downdrafts and updrafts. The motivation is to take the asymmetry of up- and downflows created by turbulent plumes into account through an adapted closure model. We built a generalized two-scale mass-flux model (GTFM) that considers both the skew introduced by the presence of two flows and the effect of turbulence onto each flow. In order to apply the GTFM to the solar case, we introduce the plume dynamics as modelled by Rieutord \& Zahn (1995) and construct a closure model with plumes (CMP). The CMP enables to express third- and fourth-order velocity correlation products in terms of the second-order ones. When comparing with $3 \mathrm{D}$ numerical simulation results, the CMP improves the agreement for the fourth order moments in comparison with the quasi-normal approximation (QNA) or the classical mass-flux model (MFM). This excitation model reproduces the maximum of the power supplied to solar $p$ modes, when compared with GOLF observations.
\end{abstract}

Keywords. Convection, turbulence, Sun: oscillations

\section{Introduction}

In the uppermost part of the solar convective zone, turbulent entropy fluctuations and eddy motions drive acoustic oscillations. Among the different theoretical approaches, that of Samadi \& Goupil (2001) includes a detailed treatment of turbulent convection. According to this approach, the analytical expression for the acoustic power supplied to $p$ modes involves fourth-order correlation functions of the turbulent Reynolds stress and the entropy source term. We propose a new approach to build a closure model which expresses fourth-order correlation functions in terms of the second-order ones. This alternative approach consists in considering the convection zone as composed of two flows (the updrafts and downdrafts). Starting from the Gryanik \& Hartmann (2002) (hereafter GH2002) approach, we develop a generalized two-scale mass-flux model (GTFM) which takes the physical properties of each flow into account (see Sect. 2). Then a theoretical description of the plumes developed by Rieutord \& Zahn (1995) (hereafter RZ95) is used to construct the closure model with plumes (CMP) (Sect. 2). Finally, the CMP permits to compute the Reynolds stress contribution to the excitation of $p$ modes (Sect. 3). 


\section{One-point closures}

\subsection{From the QNA to the two-scale mass-flux model}

The most commonly used closure model at the level of fourth-order moments is the QNA which is valid for a Gaussian probability distribution function and was first introduced by Millionshchikov (1941). As shown by Kraichnan (1957) in the context of turbulent flows, the cumulant (which is the deviation from the QNA) can be large and therefore not negligible. Hence, one has to go further than this first order approximation.

Turbulent plumes are created at the upper boundary of the convection zone. The presence of two flows introduces an additional contribution when averaging the fluctuating quantities, since averages of fluctuating quantites over each individual flows differ from averages over the total flow. The mass-flux models (MFM) were developed in order to take this non-zero skewness into account as an alternative to the QNA (Randall et al. 1992; Gryanik \& Hartmann 2002; Canuto \& Dubovikov 1998). However, such models underestimate the fourth-order moments by as much as $70 \%$ and sometimes appear to be worse than the QNA. Therefore, they clearly miss some important physical effects present in convective flows.

Gryanik \& Hartmann (2002), hereafter GH2002, propose an interpolation between the QNA and the limit of large skewness provided by the MFM. The motivation has been to account for the fact that horizontal scales of temperature and velocity fluctuations are different (hence their improvements lead to a 'two-scale mass-flux model' (TFM)) as well as to understand and measure the effects of the skewness. However, this approach requires to solve a Reynolds stress model and fails when using the analytical expression for the skewness (Belkacem et al. 2006a; Kupka \& Robinson 2007).

\subsection{The closure model with plumes}

To obtain a semi-analytical closure model, a more realistic estimate for the skewness of velocity and temperature fluctuations is required than that provided by the MFM. It has been shown that the interpolated formula obtained by GH2002 gives a very accurate modeling of the FOM provided the skewness is taken from $3 \mathrm{D}$ numerical simulations. In Belkacem et al. (2006a) we use this expression and starting from the mass-flux decomposition we propose an exact decomposition of the third- and fourth-order moments (the GTFM). This new decomposition includes both the effect of the skew introduced by the presence of two flows and the effect of turbulence onto each flow. Thus, we use the third-order moment to obtain the skewness. With some approximations validated by the $3 \mathrm{D}$ simulations, we model the remaining terms by means of a plume model following Rieutord \& Zahn (1995).

\section{Application to stellar $p$ modes: results and conclusions}

The theoretical model of stochastic excitation considered here is basically that of Samadi \& Goupil (2001) (see also Samadi et al. 2005). It takes two sources into account that drive the resonant modes of the stellar cavity: the first one is related to the Reynolds stress tensor and the second one is caused by the advection of the turbulent fluctuations of entropy by the turbulent motions (the so-called "entropy source term"). We use the CMP to model the Reynolds stress contribution for which the fourth-order correlation product appears.

The present excitation model gives a theoretical slope of the power at intermediate and high frequencies which is in agreement with the observed data (see Fig. 1). We also find that including the CMP causes a global increase of the injected power. This brings 
the power computed with the Reynolds stress contribution alone closer to (although, at intermediate frequency, still below) the observations.

On the other hand, the power obtained by including both the Reynolds stress and the entropy fluctuation contributions reproduces the slope at low and intermediate frequencies although it slightly over-estimates the excitation rates. Note, however, that in Fig. 1 the errors bars are $1 \sigma$ error bars.

Nevertheless, various sources of uncertainties are likely to exist to explain the discrepancies at high frequency. Concerning the CMP itself, the main point is the super-adiabatic region which needs further theoretical developments to obtain a suitable closure model in this zone. Concerning the modeling of the entropy contribution itself, one must apply the CMP and to go beyond the scalar passive assumption.

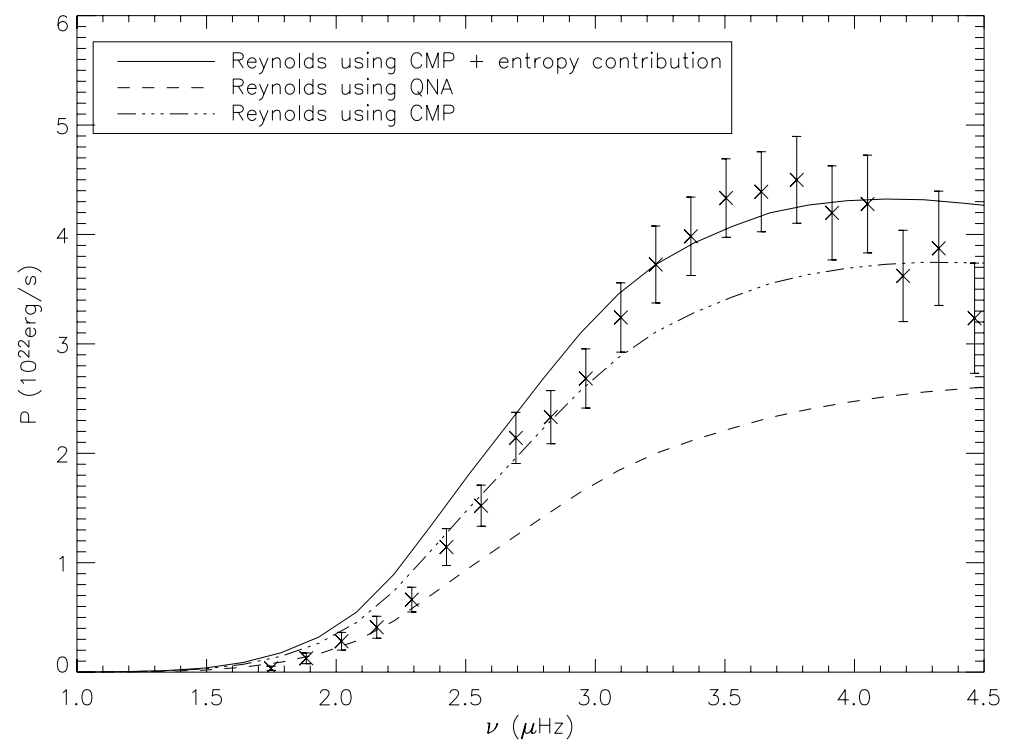

Figure 1. Rate $P$ at which acoustic energy is supplied to the solar radial modes. Cross dots represent $P$ computed from Baudin et al. (2005) solar seismic data from the GOLF instrument.

\section{References}

Abdella, K. \& McFarlane, N. 1997, J. Atmos. Sci. 54, 1850

Baudin, F., Samadi, R., Goupil, M.-J., et al. 2005, A\&ऽA 433, 349

Belkacem, K., Samadi, R.,Goupil, M.-J. \& Kupka, F., A $\& A$ 460, 173

Belkacem, K., Samadi, R.,Goupil, M.-J., Kupka, F. \& Baudin, F. A $\& A$ 460, 183

Canuto, V. M. \& Dubovikov, M. 1998, ApJ 493, 834

Gryanik, V. M. \& Hartmann, J. 2002, J. Atmos. Sci. 59, 2729

Gryanik, V. M., Hartmann, J., Raasch, S. \& Schröter, M. 2005, J. Atmos. Sci. 62, 2632

Kupka, F. \& Robinson, F. J. 2007, MNRAS 374, 305

Kraichnan, R. H. 1957, Physical Review 107, 1485

Millionshchikov, M. D. 1941, Doklady Acad. Nauk SSSR 32, 611

Randall, D. A., Shao Q. \& Moeng, C.-H. 1992, J. Atmos. Sci. 49, 1903

Rieutord, M. \& Zahn, J.-P. 1995, A 8 A 296, 127

Samadi, R. \& Goupil, M.-J. 2001, A\& A 370, 136 